\title{
Host specificity versus plasticity: testing the morphology-based taxonomy of the endoparasitic copepod family Splanchnotrophidae with COI barcoding
}

\author{
ROLAND F. ANTON ${ }^{1}$, DIRK SCHORIES ${ }^{2}$, NERIDA G. WILSON ${ }^{3,4}$, MAYA WOLF $^{5}$, MARCOS ABAD $^{6}$ \\ AND MICHAEL SCHRÖDL ${ }^{1,7,8}$ \\ ${ }^{1}$ Mollusca Department, SNSB- Bavarian State Collection of Zoology Munich, Münchhausenstraße 21, D-81247 München, Germany, \\ ${ }^{2}$ Instituto de Ciencias Marinas y Limnológicas, Universidad Austral de Chile, Valdivia, Chile, ${ }^{3}$ Molecular Systematics Unit, Western \\ Australian Museum, Welshpool, WA 6106, Australia, ${ }^{4}$ School of Animal Biology, University of Western Australia, Crawley, WA \\ 6009, Australia, ${ }^{5}$ Department of Biology, University of Oregon/Oregon Institute of Marine Biology, Charleston, OR 97420, USA, \\ ${ }^{6}$ Estación de Bioloxía Mariña da Graña, Universidade de Santiago de Compostela, Rúa da Ribeira, 1 (A Graña), 15590, Ferrol, Spain, \\ ${ }^{7}$ Department Biology II, BioZentrum, Ludwig-Maximilians-Universität München, Großhaderner Str. 2, 82152 Planegg-Martinsried, \\ Germany, ${ }^{8}$ GeoBioCenter LMU, München, Germany
}

\begin{abstract}
The Splanchnotrophidae is a family of highly modified endoparasitic copepods known to infest nudibranch or sacoglossan sea slug hosts. Most splanchnotrophid species appear to be specific to a single host, but some were reported from up to nine different host species. However, splanchnotrophid taxonomy thus far is based on external morphology, and taxonomic descriptions are, mostly, old and lack detail. They are usually based on few specimens, with intraspecific variability rarely reported. The present study used molecular data for the first time to test (1) the current taxonomic hypotheses, (2) the apparently strict host specificity of the genus Ismaila and (3) the low host specificity of the genus Splanchnotrophus with regard to the potential presence of cryptic species. Phylogenetic analyses herein used sequences of the barcoding region of the cytochrome oxidase I (COI) gene from 40 specimens representing 13 species of five genera. Species delimitation approaches include distance and barcoding gap analyses, haplotype networks and diagnostic nucleotides. Molecular results are largely compatible with the commonly accepted, morphology-based taxonomy of the Splanchnotrophidae. Strict host specificity could be confirmed for two Ismaila species. COI analyses also supported the idea that Splanchnotrophus angulatus is host-promiscuous. In Ismaila, morphology seems more suitable than barcoding to display speciation events via host switches in a recent Chilean radiation. In Splanchnotrophus, some genetic structure suggests ongoing diversification, which should be investigated further given the inadequate morphology-based taxonomy. The present study thus supports the presence of two different life history strategies in splanchnotrophids, which should be explored integratively.
\end{abstract}

Keywords: species delimitation, molecular phylogeny, DNA taxonomy, speciation, Copepoda, sea slugs, parasite

Submitted 25 June 2015; accepted 2 August 2016; first published online 13 September 2016

\section{INTRDDUCTION}

Copepods are the most abundant and speciose group in marine habitats (Yoshikoshi, 1975; Ho, 2001; Blanco-Berical et al., 2014) and they also display the greatest variety of forms (Gotto, 1979, 2004; Ho, 2001; Blanco-Berical et al., 2014). Endoparasitic copepods often exhibit extremely aberrant body forms due to the high level of adaptation to their respective host (Gotto, 1979, 2004; Huys, 2001; Haumayr \& Schrödl, 2003; Anton et al., 2015). Such is the case in Splanchnotrophidae Hancock \& Norman, 1863, a family of bizarre endoparasitic copepods exclusively infesting nudibranch and sacoglossan hosts. The family is distributed worldwide in temperate and warm coastal waters and currently

Corresponding author:

R.F. Anton

Email: rolandanton1@gmail.com comprises six genera: Splanchnotrophus Hancock \& Norman, 1863, Ismaila Bergh, 1867, Lomanoticola Scott \& Scott, 1895, Arthurius Huys, 2001, Ceratosomicola Huys, 2001 and Majimun Uyeno \& Nagasawa, 2012, with a total of now 32 species (Anton et al., 2015). All members are characterized by an enhanced body size in females, the possession of dorsal appendages (with one exception, see Anton et al., 2015), the reduction of the maxillipeds, and the abdomen of females protruding through the host's integument (Huys, 2001; Anton \& Schrödl, 2013a, b).

The taxonomy of Splanchnotrophidae is exclusively based on external morphology, with descriptions offering a highly heterogeneous level of detail and reliability. In addition, the use of external morphological characters in highly modified endoparasitic taxa has to be regarded as problematic at best (Huys, 2001). In such a case, the differentiation between true homoplasies and convergent evolution is rather complex. Most splanchnotrophids (i.e. 25 species; 78\%) are 
considered to be highly host specific, and usually each host species is infested by a single parasite species (Schrödl, 1997, 2003; Huys, 2001; Haumayr \& Schrödl, 2003; Anton \& Schrödl, 2013a, b); identification of an infested host thus may permit identification of their parasite. Interestingly, all members of the species-rich and recently reviewed genera Ismaila and Ceratosomicola are strictly host specific. A recent radiation of Chilean Ismaila species via host shifts was proposed (Schrödl, 2003; Anton \& Schrödl, 2013a, b). However, some splanchnotrophids are reported from multiple hosts (Figure $1 \mathrm{~A}$ ). The recently revised or described genera Arthurius and Majimun (Huys, 2001; Salmen et al., 2008; Uyeno \& Nagasawa, 2012) comprise a few species that are host specific and others that infest multiple host species. Similarly, five of nine species of the taxonomically obscure genera Splanchnotrophus and Lomanoticola are reported from more than one (i.e. up to nine) different species (Figure 1A) of not necessarily closely related sea slug groups (Anton \& Schrödl, 2013a). For example, Lomanoticola brevipes (Hancock \& Norman, 1863) was reported infesting members of the dexiarchian nudibranch family Dotidae, but was also found in representatives of the aeolid families Flabellinidae, Tergipedidae, Facelinidae and Eubranchidae. Different splanchnotrophid genera and species thus display different patterns of host specificity, possibly reflecting phylogenetic constraints on their ability to detect, colonize or survive in different hosts (Anton \& Schrödl, 2013a, b). It is also striking that five of the seven splanchnotrophids known from more than one host species occur exclusively in the Mediterranean Sea and along the European coasts of the Atlantic ocean (Figure $1 \mathrm{~B}$ ). These areas are among the earliest and most intensely studied with regard to marine invertebrates. However, neither the parasites nor their hosts are of apparent commercial value, and original or subsequent descriptions of European splanchnotrophids are typically old and usually based on single individuals with no adequate vouchers deposited for later study (Canu, 1891; Hecht, 1895; Bassett-Smith, 1903; O'Donoghue, 1924; Delamare Deboutteville, 1950).

Estimates of host specificity in splanchnotrophid copepods, and conclusions on the presence, ecology and evolution of highly heterogeneous specificity in different genera and geographic areas entirely depend on taxonomic identifications of parasites and hosts. On the host side, taxonomy appears straightforward, although the existence of cryptic species has only been tested by molecular data for two complexes. Both the Cratena peregrina (Gmelin, 1791) (Padula et al., 2014) and the Spurilla neapolitana (Delle Chiaje, 1841) (Carmona et al., 2014) complexes were split up using integrative taxonomic evidence. To date, splanchnotrophid taxonomy is exclusively based on (external) morphology, and little is known about intrapopulational variation (Anton \& Schrödl, 2013a, b); taxonomically relevant features such as special details of mouth parts are unknown for several species, i.e. several but not all of the species described to inhabit different hosts (Huys, 2001; Haumayr \& Schrödl, 2003; Anton \& Schrödl, 2013a, b). In general, the morphology of endoparasites can be especially adapted to their environment, i.e. conditions in their hosts (Gotto, 1979; Huys, 2001). For example, large-sized hosts may allow for longer body lengths, and the morphology of the host may affect the position of the parasites inside the hosts. Therefore, it is a crucial task to evaluate phenotypic splanchnotrophid taxonomy using genetic data, testing the assumption of narrowly adapted parasite species against host-induced plasticity. Anton \& Schrödl (2013a, b) provided a morphocladistic hypothesis on the phylogeny of splanchnotrophids and also proposed a preliminary scenario of character evolution and coevolution of splanchnotrophids with certain host groups. Since parts of the tree were not robustly supported, investigating historic and recent coevolution requires molecular analyses. DNA sequence data for splanchnotrophids has been lacking entirely, due to the difficulty of collecting and preserving a variety of rare or at least sporadic endoparasites.

The present study for the first time uses molecular data to (1) test the current taxonomic hypotheses on Splanchnotrophidae introduced by Huys (2001) and recently confirmed by morphocladistic analysis (Anton \& Schrödl, 2013a, b); (2) test the strict host specificity reported for the genus Ismaila (potentially leading to the highest species diversity of all splanchnotrophid genera) against undiscovered hostinduced phenotypic plasticity; and (3) evaluate the supposedly low host specificity of Splanchnotrophus against the possibility of the presence of cryptic species.

To test general taxonomic hypotheses, phylogenetic analyses were conducted, using 38 novel barcode sequences of the cytochrome oxidase I (COI) gene from 12 morphospecies, covering four splanchnotrophid genera. To further study host specificity, species delimitation analyses were performed focusing on two supposedly strictly host-specific species of Ismaila (Ismaila aliena Haumayr \& Schrödl, 2003, Ismaila robusta Haumayr \& Schrödl, 2003) and on Splanchnotrophus angulatus Hecht, 1893, a species currently known from five different host species. Here, a variety of molecular methods complement and extend the traditional view on species boundaries in splanchnotrophids, and allows for a preliminary integrative view on life history traits such as host specificity.

\section{MATERIALS AND METHODS}

\section{Species sampling}

For molecular analyses all ethanol-fixed splanchnotrophid samples available in the collection of the Bavarian State Collection of Zoology (ZSM) were used to obtain genetic material. Additional samples of I. aliena, I. robusta and S. angulatus were gathered during several collection trips to southern Chile in 2008 and 2010, and to southern France in 2010. Wherever possible, egg sacs were carefully removed from the host using forceps as soon as possible after collection. Samples were then stored in 96\% ethanol and kept chilled until the DNA extraction was performed. A detailed list of all included specimens is given in Table 1.

\section{DNA extraction, amplification and sequencing}

We used a NucleoSpin Tissue Kit (Macherey-Nagel, Düren, Germany) and extraction procedures followed manufacturers' instructions. Universal primers LCO-1490 (forward) and HCO-2198 (reverse) (Folmer et al., 1994) were used to amplify a $\sim 650 \mathrm{bp}$ segment of the cytochrome oxidase I (COI) gene. For amplification Illustra PuRe Taq Ready-ToGo PCR beads (GE Healthcare) were used. A mix of $0.5 \mu \mathrm{l}$ of each primer (conc. $10 \mathrm{pm}$, Metabion) plus $23 \mu \mathrm{l}$ of molecular water was added to $1.0 \mu \mathrm{l}$ of raw DNA. For PCR conditions 

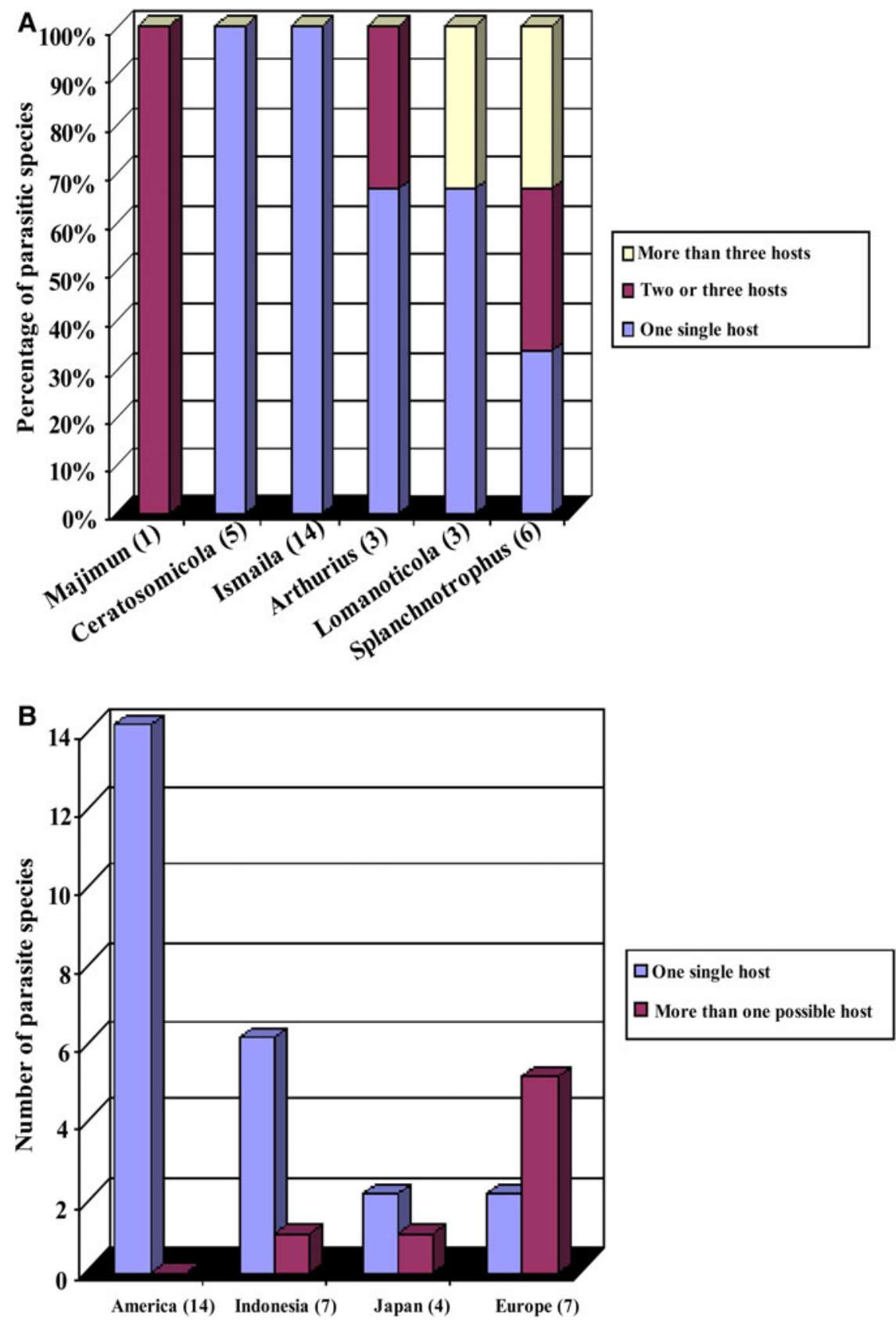

Fig. 1. Overview of the number of host species (A) per splanchnotrophid genus and (B) given the geographic distribution area following Anton \& Schrödl (2013a, b). Total number of species given in parentheses.

we applied $94^{\circ} \mathrm{C}-300 \mathrm{~s}$ for the initial step, then $94^{\circ} \mathrm{C}-45 \mathrm{~s}$, $45^{\circ} \mathrm{C}-50 \mathrm{~s}, 72^{\circ} \mathrm{C}-200 \mathrm{~s}$ for 40 cycles, with a final elongation of $72^{\circ} \mathrm{C}-600 \mathrm{~s}$. For purification of the PCR-product a NucleoSpin Extract II kit (Macherey-Nagel, Düren, Germany) was used following the manufacturer's instructions. The complete sequencing process was carried out on an $\mathrm{ABI}$ 373048 capillary sequencer by the Sequencing Service Unit of the Ludwig-Maximilians-University Munich. All sequence amplicons were subjected to a nucleotide BLAST search to test for contamination.

\section{Phylogenetic analysis}

COI fragments of 38 splanchnotrophid specimens $(12$ species from four genera) were obtained. Outgroups included
Pionodesmotes domhainfharraigeanus Anton, Stevenson \& Schwabe, 2013 (GenBank accession no. KF652042) and Cyclopoida sp. (JX948803.1) (see also Table 1). Consensus sequences were generated with BIOEDIT (Hall, 1999), edited, translated into amino acid sequences using the invertebrate mitochondrial genetic code, checked for stop codons and frame shifts, and aligned with Muscle using the MEGA 5.0 software (Tamura et al., 2011). The alignment then was masked by GBlocks (Castresana, 2000; Talavera \& Castresana, 2007) applying less stringent options; substitutional saturation was statistically tested using DAmBE (Xia et al., 2003; Xia \& Lemey, 2009); base pair frequencies and p-distances were calculated with MEGA 5.0.

A maximum likelihood (ML) analysis with 1000 bootstrap (BS) replicates was conducted with RAxML (Stamatakis, 
Table 1. Overview of all included specimens giving the registration number, the host specimens and the country and exact location of the collection site respectively.

\begin{tabular}{|c|c|c|c|c|c|c|c|c|c|}
\hline Voucher ID & $\begin{array}{l}\text { GenBank } \\
\text { accession } \\
\text { number }\end{array}$ & Species & ZSM-ID & Host & ZSM-ID & Country/Region & Latitude & Longitude & Depth (m) \\
\hline G 001 & $\mathrm{KT}_{122805}$ & Splanchnotrophus angulatus & ZSMA20142906 & Flabellina ischitana & ZSM-Mol10100477 & Southern France/Banyuls & $42^{\circ} 28^{\prime} 56.20^{\prime \prime} \mathrm{N}$ & $3^{\circ} 08^{\prime} 13.19^{\prime \prime} \mathrm{O}$ & $2-5$ \\
\hline G o02 & KT122806 & Splanchnotrophus angulatus & ZSMA20142907 & Spurilla neapolitana & ZSM-Mol20100409 & Croatia/Mala Portic & $44^{\circ} 46^{\prime} 45.15^{\prime \prime} \mathrm{N}$ & $13^{\circ} 55^{\prime} 10.84^{\prime \prime} \mathrm{O}$ & $2-5$ \\
\hline $\mathrm{G} \mathrm{o03}$ & $\mathrm{KT}_{122807}$ & Splanchnotrophus angulatus & ZSMA20142908 & Spurilla neapolitana & ZSM-Mol20100409 & Croatia/Mala Portic & $44^{\circ} 46^{\prime} 45.15^{\prime \prime} \mathrm{N}$ & $13^{\circ} 55^{\prime} 10.84^{\prime \prime} \mathrm{O}$ & $2-5$ \\
\hline G o04 & KT122808 & Splanchnotrophus angulatus & ZSMA20142909 & Cratena peregrina & ZSM-Mol20130874 & Islote 5.6.1998 & & & \\
\hline G 005 & $\mathrm{KT}_{122809}$ & Splanchnotrophus angulatus & ZSMA20142910 & Aeolidiella alderi & ZSM-Mol20070272 & & & & \\
\hline G oo6 & KT122810 & Ismaila robusta & inside host & Phidiana lottini & ZSM-Mol20110432 & Southern Chile/Playa Chica & $39^{\circ} 43^{\prime} 10^{\prime \prime} \mathrm{S}$ & $73^{\circ} 24^{\prime} 12^{\prime \prime} \mathrm{W}$ & 2 \\
\hline Go11 & $\mathrm{KT} 122812$ & Splanchnotrophus angulatus & ZSMA20142912 & Cratena peregrina & host lost & Southern France/Banyuls & $42^{\circ} 28^{\prime} 56.20^{\prime \prime} \mathrm{N}$ & $3^{\circ} 08^{\prime} 13.19^{\prime \prime} \mathrm{O}$ & $2-5$ \\
\hline G 012 & $\mathrm{KT}_{122813}$ & Splanchnotrophus angulatus & inside host & Cratena peregrina & ZSM-Mol20130849 & Southern France/Banyuls & $42^{\circ} 28^{\prime} 56.20^{\prime \prime} \mathrm{N}$ & $3^{\circ} 08^{\prime} 13.19^{\prime \prime} \mathrm{O}$ & $2-5$ \\
\hline $\mathrm{G} 013$ & $\mathrm{KT}_{122814}$ & Ismaila aliena & inside host & Thecacera darwini & ZSM-Mol20130850 & Southern Chile/Valdivia & $39^{\circ} 57^{\prime} 25.94^{\prime \prime} \mathrm{S}$ & $73^{\circ} 36^{\prime} 10.15^{\prime \prime} \mathrm{W}$ & $6-10$ \\
\hline G 015 & $\mathrm{KT}_{122815}$ & Ismaila genalis & ZSMA20142903 & Holoplocamus papposus & ZSM-Mol20130872 & Southern Chile/Isla Carmen & $43^{\circ} \mathrm{O1}^{\prime} \mathrm{O} 8.8 \mathrm{o}^{\prime \prime} \mathrm{S}$ & $72^{\circ} 49^{\prime} 44.79^{\prime \prime} \mathrm{W}$ & $1-20$ \\
\hline G 016 & $\mathrm{KT}_{122816}$ & Ismaila belciki & ZSMA20142916 & Janolus fuscus & host lost & USA/Oregon & $43^{\circ} 21^{\prime} 32.4^{\prime \prime} \mathrm{N}$ & $124^{\circ} 18^{\prime} 45.36^{\prime \prime} \mathrm{W}$ & $0-2$ \\
\hline $\mathrm{G} 017$ & $\mathrm{KT}_{122817}$ & Ismaila volatilis & ZSMA20142900 & Janolus spec. & ZSM-Mol20130847 & Southern Chile/Valdivia & $39^{\circ} 57^{\prime} 25.94^{\prime \prime} \mathrm{S}$ & $73^{\circ} 36^{\prime} 10.15^{\prime \prime} \mathrm{W}$ & $6-20$ \\
\hline G 019 & $\mathrm{KT}_{122818}$ & Ismaila aliena & ZSMA20142918 & Thecacera darwini & ZSM-Mol20130851 & Southern Chile/Valdivia & $39^{\circ} 57^{\prime} 25.94^{\prime \prime} \mathrm{S}$ & $73^{\circ} 36^{\prime} 10.15^{\prime \prime} \mathrm{W}$ & $6-10$ \\
\hline $\mathrm{G} 020$ & $\mathrm{KT}_{122819}$ & Ismaila aliena & ZSMA20142919 & Thecacera darwini & ZSM-Mol20130851 & Southern Chile/Valdivia & $39^{\circ} 57^{\prime} 25.94^{\prime \prime} \mathrm{S}$ & $73^{\circ} 36^{\prime} 10.15^{\prime \prime} \mathrm{W}$ & $6-10$ \\
\hline G 021 & $\mathrm{KT} 122820$ & Ismaila aliena & inside host & Thecacera darwini & ZSM-Mol20130852 & Southern Chile/Valdivia & $39^{\circ} 57^{\prime} 25.94^{\prime \prime} \mathrm{S}$ & $73^{\circ} 36^{\prime} 10.15^{\prime \prime} \mathrm{W}$ & $6-10$ \\
\hline G 022 & $\mathrm{KT}_{122821}$ & Ismaila robusta & ZSMA20142921 & Phidiana lottini & host lost & Southern Chile/Valdivia & $39^{\circ} 57^{\prime} 25.94^{\prime \prime S}$ & $73^{\circ} 36^{\prime} 10.15^{\prime \prime} \mathrm{W}$ & $6-10$ \\
\hline $\mathrm{G} 023$ & $\mathrm{KT}_{122822}$ & Ismaila robusta & ZSMA20142921 & Phidiana lottini & host lost & Southern Chile/Valdivia & $39^{\circ} 57^{\prime} 25.94^{\prime \prime} \mathrm{S}$ & $73^{\circ} 36^{\prime} 10.15^{\prime \prime} \mathrm{W}$ & $6-10$ \\
\hline G 024 & $\mathrm{KT}_{122823}$ & Ismaila robusta & ZSMA20142923 & Phidiana lottini & host lost & Southern Chile/Valdivia & $39^{\circ} 57^{\prime} 25.94^{\prime \prime} \mathrm{S}$ & $73^{\circ} 36^{\prime} 10.15^{\prime \prime} \mathrm{W}$ & $6-10$ \\
\hline G 025 & KT122824 & Splanchnotrophus angulatus & inside host & Spurilla neapolitana & ZSM-Mol20110684 & Italy/Bastione Conca & $38^{\circ} 01^{\prime} 03^{\prime \prime} \mathrm{N}$ & $12^{\circ} 30^{\prime} 14^{\prime \prime} \mathrm{E}$ & $2-5$ \\
\hline G 028 & $\mathrm{KT}_{122825}$ & Ismaila robusta & ZSMA20142925 & Phidiana lottini & ZSM-Mol20130855 & Southern Chile/Valdivia & $39^{\circ} 57^{\prime} 25.94^{\prime \prime} \mathrm{S}$ & $73^{\circ} 36^{\prime} 10.15^{\prime \prime} \mathrm{W}$ & $6-10$ \\
\hline G 029 & $\mathrm{KT}_{122826}$ & Ismaila aliena & inside host & Thecacera darwini & ZSM-Mol20130856 & Southern Chile/Valdivia & $39^{\circ} 57^{\prime} 25.94^{\prime \prime} S$ & $73^{\circ} 36^{\prime} 10.15^{\prime \prime} \mathrm{W}$ & $6-10$ \\
\hline G o3o & $\mathrm{KT}_{122827}$ & Ismaila aliena & inside host & Thecacera darwini & ZSM-Mol20130856 & Southern Chile/Valdivia & $39^{\circ} 57^{\prime} 25.94^{\prime \prime} \mathrm{S}$ & $73^{\circ} 36^{\prime} 10.15^{\prime \prime} \mathrm{W}$ & $6-10$ \\
\hline G 031 & $\mathrm{KT}_{122828}$ & Ismaila aliena & inside host & Thecacera darwini & ZSM-Mol20130857 & Southern Chile/Valdivia & $39^{\circ} 57^{\prime} 25.94^{\prime \prime S}$ & $73^{\circ} 36^{\prime} 10.15^{\prime \prime} \mathrm{W}$ & $6-10$ \\
\hline $\mathrm{G} \mathrm{O32}$ & $\mathrm{KT}_{122829}$ & Ismaila chaihuiensis & ZSMA20142902 & Diaulula punctuolata & ZSM-Mol20130858 & Southern Chile/Valdivia & $39^{\circ} 57^{\prime} 25.94^{\prime \prime} \mathrm{S}$ & $73^{\circ} 36^{\prime} 10.15^{\prime \prime} \mathrm{W}$ & $6-10$ \\
\hline G o34 & $\mathrm{KT} 122830$ & Ismaila damnosa & ZSMA20142905 & Flabellina sp. 1 & host lost & Southern Chile/Valdivia & $39^{\circ} 57^{\prime} 25.94^{\prime \prime} \mathrm{S}$ & $73^{\circ} 36^{\prime} 10.15^{\prime \prime} \mathrm{W}$ & 12 \\
\hline G 035 & KT122831 & Splanchnotrophus angulatus & inside host & Cratena peregrina & ZSM-Mol20130860 & Southern France/Banyuls & $42^{\circ} 28^{\prime} 56.20^{\prime \prime} \mathrm{N}$ & $3^{\circ} 08^{\prime} 13.19^{\prime \prime} \mathrm{O}$ & $2-5$ \\
\hline $\mathrm{G} \mathrm{o36}$ & $\mathrm{KT}_{122832}$ & Splanchnotrophus angulatus & ZSMA20142930 & Cratena peregrina & host lost & Southern France/Banyuls & $42^{\circ} 28^{\prime} 56.20^{\prime \prime} \mathrm{N}$ & $3^{\circ} 08^{\prime} 13.19^{\prime \prime} \mathrm{O}$ & $2-5$ \\
\hline G 038 & $\mathrm{KT}_{122833}$ & Lomanoticola spec. & ZSMA20142931 & Cuthona cerulea & ZSM-Mol20130862 & Southern France/Banyuls & $42^{\circ} 28^{\prime} 56.20^{\prime \prime} \mathrm{N}$ & $3^{\circ} 08^{\prime} 13.19^{\prime \prime} \mathrm{O}$ & $2-5$ \\
\hline $\mathrm{G} \mathrm{o42}$ & KF652042 & $\begin{array}{l}\text { Pionodesmotes } \\
\quad \text { domhainfharraigeanus }\end{array}$ & ZSMA20130004 & Sperosoma grimaldii & host lost & Ireland/Whittard Canyon & $48.491^{\circ} \mathrm{N}$ & $10.692^{\circ} \mathrm{W}$ & 2000 \\
\hline G 044 & $\mathrm{KT}_{122834}$ & Ceratosomicola mammilata & inside host & $\begin{array}{r}\text { Chromodoris } \\
\text { geometrica }\end{array}$ & ZSM-Mol20130863 & Indonesia/Sulawesi & $5^{\circ} 28^{\prime} 29^{\prime \prime} \mathrm{S}$ & $123^{\circ} 45^{\prime} 40^{\prime \prime} \mathrm{E}$ & 4 \\
\hline $\mathrm{G} 046$ & $\mathrm{KT} 122835$ & Splanchnotrophus gracilis & ZSMA20142933 & Trapania tartanella & host lost & Spain/Ria de Ferrol & $43^{\circ} 28^{\prime} 02.16^{\prime \prime} \mathrm{N}$ & $8^{\circ} 14^{\prime} 47.70^{\prime \prime} \mathrm{W}$ & 20 \\
\hline G 055 & $\mathrm{KT} 122836$ & Splanchnotrophus angulatus & inside host & Cratena peregrina & ZSM-Mol20130864 & Southern France/Banyuls & $42^{\circ} 28^{\prime} 56.20^{\prime \prime} \mathrm{N}$ & $3^{\circ} 08^{\prime} 13.19^{\prime \prime} \mathrm{O}$ & $2-5$ \\
\hline G 056 & KT122837 & Splanchnotrophus angulatus & ZSMA20142935 & Cratena peregrina & ZSM-Mol20130865 & Southern France/Banyuls & $42^{\circ} 28^{\prime} 56.20^{\prime \prime} \mathrm{N}$ & $3^{\circ} 08^{\prime} 13.19^{\prime \prime} \mathrm{O}$ & $2-5$ \\
\hline G 057 & KT122838 & Ismaila robusta & ZSMA20142936 & Phidiana lottini & host lost & Southern Chile/Valdivia & $39^{\circ} 57^{\prime} 25.94^{\prime \prime} \mathrm{S}$ & $73^{\circ} 36^{\prime} 10.15^{\prime \prime} \mathrm{W}$ & $6-10$ \\
\hline G 058 & KT122839 & Splanchnotrophus angulatus & inside host & Cratena peregrina & ZSM-Mol20130867 & Southern France/Banyuls & $42^{\circ} 28^{\prime} 56.20^{\prime \prime} \mathrm{N}$ & $3^{\circ} 08^{\prime} 13.19^{\prime \prime} \mathrm{O}$ & $2-5$ \\
\hline G 059 & $\mathrm{KT}_{122840}$ & Ismaila robusta & ZSMA20142938 & Phidiana lottini & ZSM-Mol20130868 & Southern Chile/Valdivia & $39^{\circ} 57^{\prime} 25.94^{\prime \prime} \mathrm{S}$ & $73^{\circ} 36^{\prime} 10.15^{\prime \prime} \mathrm{W}$ & $6-10$ \\
\hline G 060 & $\mathrm{KT} 122841$ & Ismaila robusta & inside host & Phidiana lottini & ZSM-Mol20130869 & Southern Chile/Valdivia & $39^{\circ} 57^{\prime} 25.94^{\prime \prime} \mathrm{S}$ & $73^{\circ} 36^{\prime} 10.15^{\prime \prime} \mathrm{W}$ & $6-10$ \\
\hline G 082 & $\mathrm{KT}_{122842}$ & Ismaila volatilis & inside host & Janolus sp. & ZSM-Mol20130866 & Southern Chile/Valdivia & $39^{\circ} 57^{\prime} 25.94^{\prime \prime} \mathrm{S}$ & $73^{\circ} 36^{\prime} 10.15^{\prime \prime} \mathrm{W}$ & $6-20$ \\
\hline G 100 & $\mathrm{KT}_{122811}$ & Ismaila spec. & inside host & Eubranchus sp. 2 & ZSM-Mol20130871 & Southern Chile/Isla Traiguen & $45^{\circ} 11^{\prime} 26.11^{\prime \prime} \mathrm{S}$ & $73^{\circ} 30^{\prime} 49.69^{\prime \prime} \mathrm{W}$ & 6 \\
\hline
\end{tabular}


2014) using the GTRCAT model. Bayesian inference (BI) with MrBAYES (Ronquist \& Huelsenbeck, 2003) used the invertebrate mitochondrial code, the codon nucleotide model, and 2 million generations, with a sampling frequency of 500 generations. In addition neighbour network graphs were calculated using SplitsTree4 (Huson \& Bryant, 2006) to check for incompatibilities within the data.

\section{Detection of barcode gaps, haplotype networks and diagnostic nucleotides}

For the genera Splanchnotrophus and Ismaila a search for barcode gaps was performed using alignments of all sequences of the respective genera and the ABGD-software (Puillandre et al., 2011, 2012), which sorts the sequences into hypothetical species based on the barcode gap, which can be observed whenever the divergence among organisms belonging to the same species is smaller than divergence among organisms from different species. A second approach, Species IDENTIFIER (Meier et al., 2006), was used to calculate pairwise distances (see Table 2) and clusters that identify potential species. A third approach was also used, a Poisson Tree Processes (PTP) model (Zhang et al., 2013) provided on the webserver of The Exelixis Lab (URL: http://sco.h-its.org/exelixis/web/ software/PTP/index.html), with default settings of 100,000 MCMC generations and a burn-in of 0.1. Furthermore, a statistical parsimony network was conducted on all 13 sequences of $S$. angulatus and on the 19 sequences representing the genus Ismaila using the TCs 1.2 software (Clement et al., 2009). Diagnostic characters were obtained through searching the overall alignment following the definition given by Sarkar et al. (2008) for single pure and single private characters.

\section{RESULTS}

\section{Phylogenetic hypothesis}

The final COI alignment consisted of $615 \mathrm{bp}$, including 38 splanchnotrophid specimens (12 morphologically defined species from four genera) and two outgroup taxa. In Splanchnotrophidae, the mean base pair frequencies for $\mathrm{T}$ (34.8\%), C (19.5\%), A (25.2\%) and G (20.5\%) reflected the bias towards adenosine and thymine which is characteristic for arthropods (Weis \& Melzer, 2012). The index of substitution saturation (Iss) was tested for the whole alignment after Xia \& Lemey (2009) with an estimated proportion of invariant sites of 0.54; this was significantly lower than the critical Iss.c value, indicating no substitutional saturation.

Although the neighbour network built with the SPLITSTREE4 software revealed some conflict within the clades of Ismaila and $S$. angulatus, there were very few incompatible splits within the data (Figure 2A). Regarding Splanchnotrophus, the specimens parasitizing the nudibranchs $S$. neapolitana and $A$. alderi were recovered as strictly separated to a group including all those utilizing C. peregrina or F. ischitana as hosts (see Figure $2 \mathrm{~B}$ ). On the other hand, I. belciki was recovered as the most basal sister taxon to all other members of the genus. In addition there was split support for a group comprising I. volatilis, Ismaila sp. and I. damnosa, with I. chaihuiensis as a basal offshoot (see Figure $2 \mathrm{C}$ ).
Both ML and BI analyses led to two similar trees, only differing in two regions. In both analyses the Splanchnotrophidae are recovered as a clade with high support (BS 100/ BI 1). Ceratosomicola mammilata Salmen, Wilson \& Schrödl, 2008 formed the highly supported (BS 100/BI 1) sistergroup to the rest, followed by Splanchnotrophus gracilis Hancock \& Norman, 1863; then all members of S. angulatus was recovered as the sister clade to a poorly supported clade formed by Lomanoticola and the monophyletic genus Ismaila (BS 100/BI 1). Inside the monophyletic (BS 100/BI 1) S. angulatus most of the sequences from specimens found in the aeolid nudibranch host Cratena peregrina (Facelinidae) clustered together with one sequence from a specimen extracted from the aeolid Flabellina ischitana Hirano \& Thompson, 1990 (Flabellinidae). However in the ML analysis the clade resulted as a trichotomy consisting of sequence $\mathrm{G}_{11}$, a clade comprising of the three sequences Go2, G03 and G25 (infesting the aeolid Spurilla neapolitana; Aeolidiidae) together with the sequence G05 (infesting Aeolidiella alderi (Cocks, 1852); Aeolidiidae) and a clade with the rest of the sequences as described above (Figure 3). In contrast, the BI analysis recovered a subclade consisting of the sequences Go2, Go3, G05, G11 and G25 originating from a polytomy formed by the rest of the sequences as described above (Figure 4).

The topologies recovered for the Ismaila clade were similar in both analyses with I. aliena and I. robusta both strongly supported individually and as a sister group. However, the results of the ML analysis suggested a clade with Ismaila chaihuiensis Anton, Schories, Jörger, Kalagis \& Schrödl, 2015 as its most basal offshoot to a dichotomy of a clade consisting of undescribed Ismaila sp. and Ismaila damnosa Haumayr \& Schrödl, 2003 and a clade comprising Ismaila volatilis Anton et al., 2015 and Ismaila genalis Anton et al., 2015, forming the sister to the clade of I. aliena and I. robusta (Figure 3), but with only low support values. In contrast, BI favoured a polytomy of I. volatilis, I. genalis, a clade comprising I. damnosa and Ismaila sp. and a dichotomy of I. aliena and $I$. robusta. Within I. robusta three sequences (G22, G24 \& G28) formed a subclade with moderate support (BS62/ BI96, see also Figure 4) in both analyses.

\section{Distances and barcode gaps}

P-distances between the included splanchnotrophid genera are given in Table 2. Within genera the ABGD-analyses revealed strong barcode gaps. In Ismaila, ABGD favoured five groups: group 1 consists of Ismaila sp., I. genalis, I. volatilis and I. damnosa; group 2 represents I. robusta; group 3 represents I. aliena; group 4 I. belciki and group 5 I. chaihuiensis. For the genus Splanchnotrophus the ABGD-analyses also revealed a strong barcode gap between $S$. angulatus and S. gracilis, but between $P=0.0010$ and $P=0.0046$ the sequences formed three different groups, with two sequences separated from the rest of the $S$. angulatus group. Excluding S. gracilis, ABGD still favoured this split within S. angulatus; however, there is no clear detectable barcode gap.

The software SPECIES IDENTIFIER found 12 clusters, under a threshold of $2.42 \%$, calculated from a pairwise summary. Clusters 1 and 10 represent the two outgroup taxa. Cluster 2 included all S. angulatus sequences and cluster 3 represented I. robusta. Cluster 4 included all sequences of I. volatilis, Ismaila sp. and I. damnosa. Clusters 5, 6, 7, 8 and 9 represented the species I. aliena, I. genalis, I. belciki, I. chaihuiensis 
Table 2. Data output of pairwise distances calculated with SPECIES IDENTIFIER.

\begin{tabular}{|c|c|c|c|c|c|c|}
\hline Sequence name & Largest conspecific match & Distance & Overlap & $\begin{array}{l}\text { Closest congeneric. } \\
\text { interspecific match }\end{array}$ & Distance & Overlap \\
\hline Cyclopoida sp. & $\begin{array}{l}\text { No matching conspecific } \\
\text { sequence }\end{array}$ & N/A & N/A & $\begin{array}{l}\text { No matching congeneric, } \\
\text { interspecific sequence }\end{array}$ & N/A & N/A \\
\hline Go1 Splanchnotrophus angulatus F & $\begin{array}{l}\text { Go3 Splanchnotrophus } \\
\text { angulatus S }\end{array}$ & 2.19 & 638 & G46 Splanchnotrophus gracilis & 15.55 & 643 \\
\hline Go2 Splanchnotrophus angulatus S & $\begin{array}{l}\text { G12 Splanchnotrophus } \\
\text { angulatus } \mathrm{C}\end{array}$ & 2.73 & 657 & G46 Splanchnotrophus gracilis & 16.19 & 667 \\
\hline Go3 Splanchnotrophus angulatus S & $\begin{array}{l}\text { G12 Splanchnotrophus } \\
\text { angulatus } \mathrm{C}\end{array}$ & 2.73 & 657 & G46 Splanchnotrophus gracilis & 16.46 & 662 \\
\hline Go4 Splanchnotrophus angulatus C & $\begin{array}{l}\text { Go3 Splanchnotrophus } \\
\text { angulatus S }\end{array}$ & 2.42 & 660 & G46 Splanchnotrophus gracilis & 15.6 & 660 \\
\hline Go5 Splanchnotrophus angulatus A & $\begin{array}{l}\text { Go3 Splanchnotrophus } \\
\text { angulatus S }\end{array}$ & 1.81 & 662 & G46 Splanchnotrophus gracilis & 14.39 & 667 \\
\hline Go6 Ismaila robusta & $\mathrm{G}_{57}$ Ismaila robusta & 0.62 & 639 & G13 Ismaila aliena & 4.06 & 639 \\
\hline G1oo Ismaila sp. & $\begin{array}{l}\text { No matching conspecific } \\
\text { sequence }\end{array}$ & N/A & N/A & G34 Ismaila damnosa & 1.48 & 672 \\
\hline G11 Splanchnotrophus angulatus C & $\begin{array}{l}\text { Go3 Splanchnotrophus } \\
\text { angulatus S }\end{array}$ & 2.11 & 662 & G46 Splanchnotrophus gracilis & 15.14 & 667 \\
\hline G12 Splanchnotrophus angulatus C & $\begin{array}{l}\text { Go3 Splanchnotrophus } \\
\text { angulatus S }\end{array}$ & 2.73 & 657 & G46 Splanchnotrophus gracilis & 15.67 & 657 \\
\hline G13 Ismaila aliena & G21 Ismaila aliena & 0.44 & 671 & G23 Ismaila robusta & 4.01 & 672 \\
\hline G15 Ismaila genalis & $\begin{array}{l}\text { No matching conspecific } \\
\text { sequence }\end{array}$ & N/A & N/A & Go6 Ismaila robusta & 5.63 & 639 \\
\hline G16 Ismaila belciki & $\begin{array}{l}\text { No matching conspecific } \\
\text { sequence }\end{array}$ & $\mathrm{N} / \mathrm{A}$ & N/A & G10o Ismaila sp. & 12.2 & 672 \\
\hline G17 Ismaila volatilis & G82 Ismaila volatilis & 2.1 & 666 & G34 Ismaila damnosa & 1.63 & 671 \\
\hline G19 Ismaila aliena & G21 Ismaila aliena & 0.74 & 669 & G23 Ismaila robusta & 4.33 & 669 \\
\hline G2o Ismaila aliena & G21 Ismaila aliena & 0.59 & 671 & G23 Ismaila robusta & 4.17 & 671 \\
\hline G21 Ismaila aliena & G19 Ismaila aliena & 0.74 & 669 & G59 Ismaila robusta & 4.39 & 592 \\
\hline G22 Ismaila robusta & $\mathrm{G}_{57}$ Ismaila robusta & 0.59 & 672 & G13 Ismaila aliena & 4.16 & 672 \\
\hline G23 Ismaila robusta & $\mathrm{G}_{57}$ Ismaila robusta & 0.44 & 672 & G13 Ismaila aliena & 4.01 & 672 \\
\hline G24 Ismaila robusta & $\mathrm{G}_{57}$ Ismaila robusta & 0.89 & 671 & G13 Ismaila aliena & 4.17 & 671 \\
\hline G25 Splanchnotrophus angulatus S & $\begin{array}{l}\text { Go3 Splanchnotrophus } \\
\text { angulatus S }\end{array}$ & 2.41 & 662 & G46 Splanchnotrophus gracilis & 15.59 & 667 \\
\hline G28 Ismaila robusta & $\mathrm{G}_{57}$ Ismaila robusta & 0.74 & 672 & G13 Ismaila aliena & $4 \cdot 31$ & 672 \\
\hline G29 Ismaila aliena & G21 Ismaila aliena & 0.44 & 671 & G23 Ismaila robusta & 4.01 & 672 \\
\hline G3o Ismaila aliena & G21 Ismaila aliena & 0.44 & 671 & G23 Ismaila robusta & 4.01 & 672 \\
\hline G31 Ismaila aliena & G21 Ismaila aliena & 0.74 & 670 & Go6 Ismaila robusta & 4.22 & 639 \\
\hline G32 Ismaila chaihuiensis & $\begin{array}{l}\text { No matching conspecific } \\
\text { sequence }\end{array}$ & N/A & N/A & G82 Ismaila volatilis & 3.74 & 667 \\
\hline G34 Ismaila damnosa & $\begin{array}{l}\text { No matching conspecific } \\
\text { sequence }\end{array}$ & $\mathrm{N} / \mathrm{A}$ & N/A & G10o Ismaila sp. & 1.48 & 672 \\
\hline G35 Splanchnotrophus angulatus C & $\begin{array}{l}\text { Go3 Splanchnotrophus } \\
\text { angulatus S }\end{array}$ & 1.96 & 662 & G46 Splanchnotrophus gracilis & 15.14 & 667 \\
\hline $\mathrm{G}_{3} 6$ Splanchnotrophus angulatus $\mathrm{C}$ & $\begin{array}{l}\text { Go3 Splanchnotrophus } \\
\text { angulatus S }\end{array}$ & 2.11 & 662 & G46 Splanchnotrophus gracilis & 14.99 & 667 \\
\hline G38 Lomanoticola sp. & $\begin{array}{l}\text { No matching conspecific } \\
\text { sequence }\end{array}$ & N/A & N/A & $\begin{array}{c}\text { No matching congeneric, } \\
\text { interspecific sequence }\end{array}$ & N/A & N/A \\
\hline $\begin{array}{l}\text { G42 Pionodesmotes } \\
\quad \text { domhainfharraigeanus }\end{array}$ & $\begin{array}{l}\text { No matching conspecific } \\
\text { sequence }\end{array}$ & N/A & N/A & $\begin{array}{l}\text { No matching congeneric, } \\
\text { interspecific sequence }\end{array}$ & N/A & N/A \\
\hline G44 Ceratosomicola mammillata & $\begin{array}{l}\text { No matching conspecific } \\
\text { sequence }\end{array}$ & N/A & N/A & $\begin{array}{l}\text { No matching congeneric, } \\
\text { interspecific sequence }\end{array}$ & N/A & N/A \\
\hline G46 Splanchnotrophus gracilis & $\begin{array}{l}\text { No matching conspecific } \\
\text { sequence }\end{array}$ & $\mathrm{N} / \mathrm{A}$ & N/A & Go5 Splanchnotrophus angulatus A & 14.39 & 667 \\
\hline G55 Splanchnotrophus angulatus C & $\begin{array}{l}\text { Go3 Splanchnotrophus } \\
\text { angulatus S }\end{array}$ & 2.26 & 661 & G46 Splanchnotrophus gracilis & 14.86 & 666 \\
\hline G56 Splanchnotrophus angulatus C & $\begin{array}{l}\text { Go3 Splanchnotrophus } \\
\text { angulatus S }\end{array}$ & 1.96 & 662 & G46 Splanchnotrophus gracilis & 15.14 & 667 \\
\hline $\mathrm{G}_{57}$ Ismaila robusta & G24 Ismaila robusta & 0.89 & 671 & G13 Ismaila aliena & 4.46 & 672 \\
\hline G58 Splanchnotrophus angulatus C & $\begin{array}{l}\text { Go3 Splanchnotrophus } \\
\text { angulatus S }\end{array}$ & 2.43 & 658 & G46 Splanchnotrophus gracilis & 15.23 & 663 \\
\hline G59 Ismaila robusta & G24 Ismaila robusta & 0.5 & 592 & G13 Ismaila aliena & 4.22 & 592 \\
\hline G6o Ismaila robusta & $\mathrm{G}_{57}$ Ismaila robusta & 0.44 & 672 & G13 Ismaila aliena & 4.01 & 672 \\
\hline G82 Ismaila volatilis & G17 Ismaila volatilis & 2.1 & 666 & G34 Ismaila damnosa & 2.24 & 667 \\
\hline
\end{tabular}




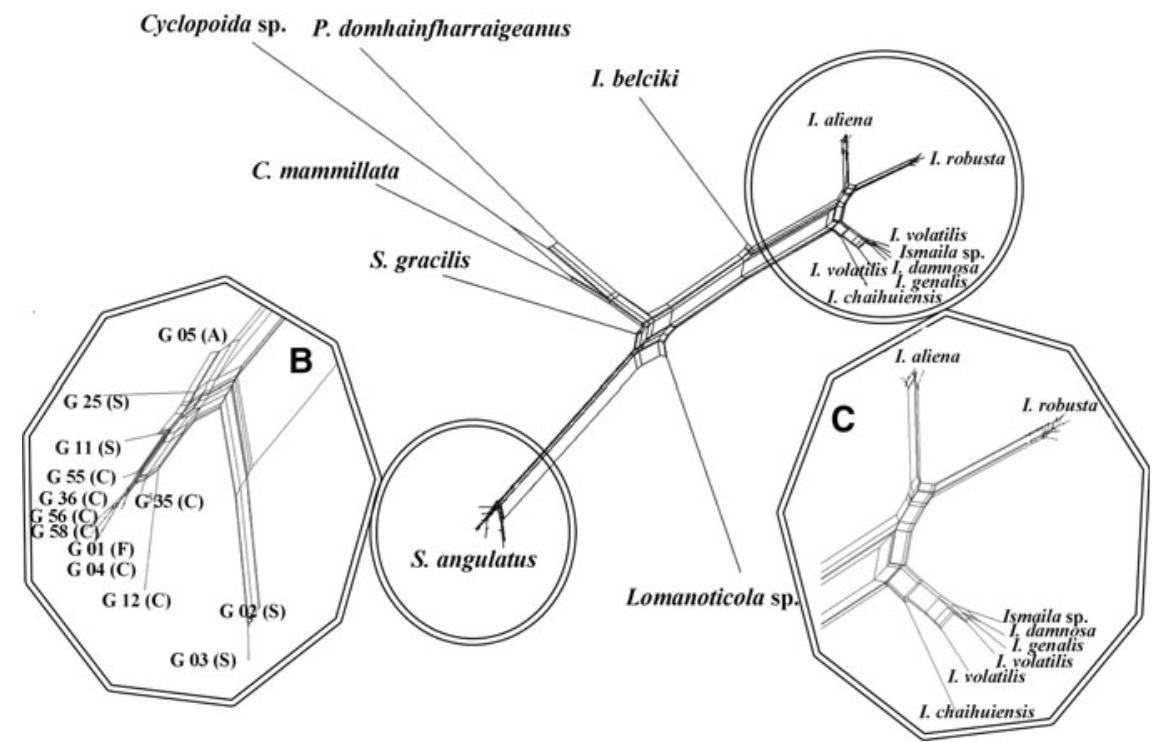

Fig. 2. Neighbour network computed by SplitsTree (A) with magnifications for the regions of interest inside (B) the Ismaila and (C) the Splanchnotrophus cluster. Capitals before or following species name refer to respective hosts: C: Cratena peregrina; S: Spurilla neapolitana; F: Flabellina ischitana; A: Aeolidiella alderi.

and Lomanoticola sp. respectively. Cluster 11 included $C$. mammillata and cluster 12 S. gracilis (see Figure $5 \mathrm{G}$ ).

The PTP-analysis indicated outgroup taxa, C. mammillata, S. gracilis, Lomanoticola sp. and I. belciki as independent species with high support values. Good support was recognized for S. angulatus, I. aliena and I. robusta. However, all recently discovered Ismaila species form one cluster, although this is poorly supported (Figure $5 \mathrm{~F}$ ). Results are mostly

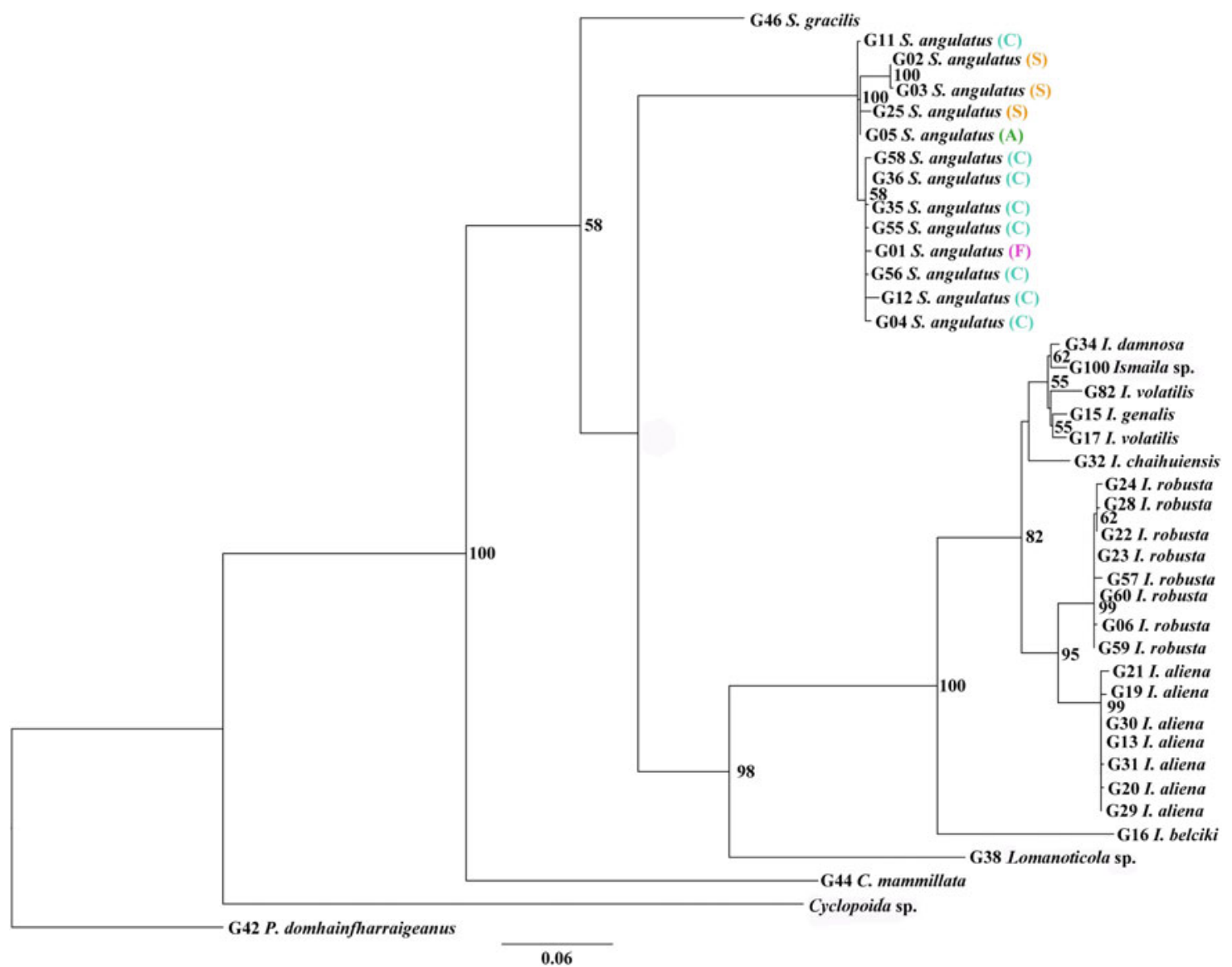

Fig. 3. Maximum likelihood consensus tree of the cytochrome c oxidase I (COI) sequences of 38 splanchnotrophids and two outgroup taxa. Numbers above branches show bootstrap values $\left(>_{55} \%\right)$; branch length indicates substitutions per site. Capitals in parentheses refer to respective hosts: C: Cratena peregrina; S: Spurilla neapolitana; F: Flabellina ischitana; A: Aeolidiella alderi. 


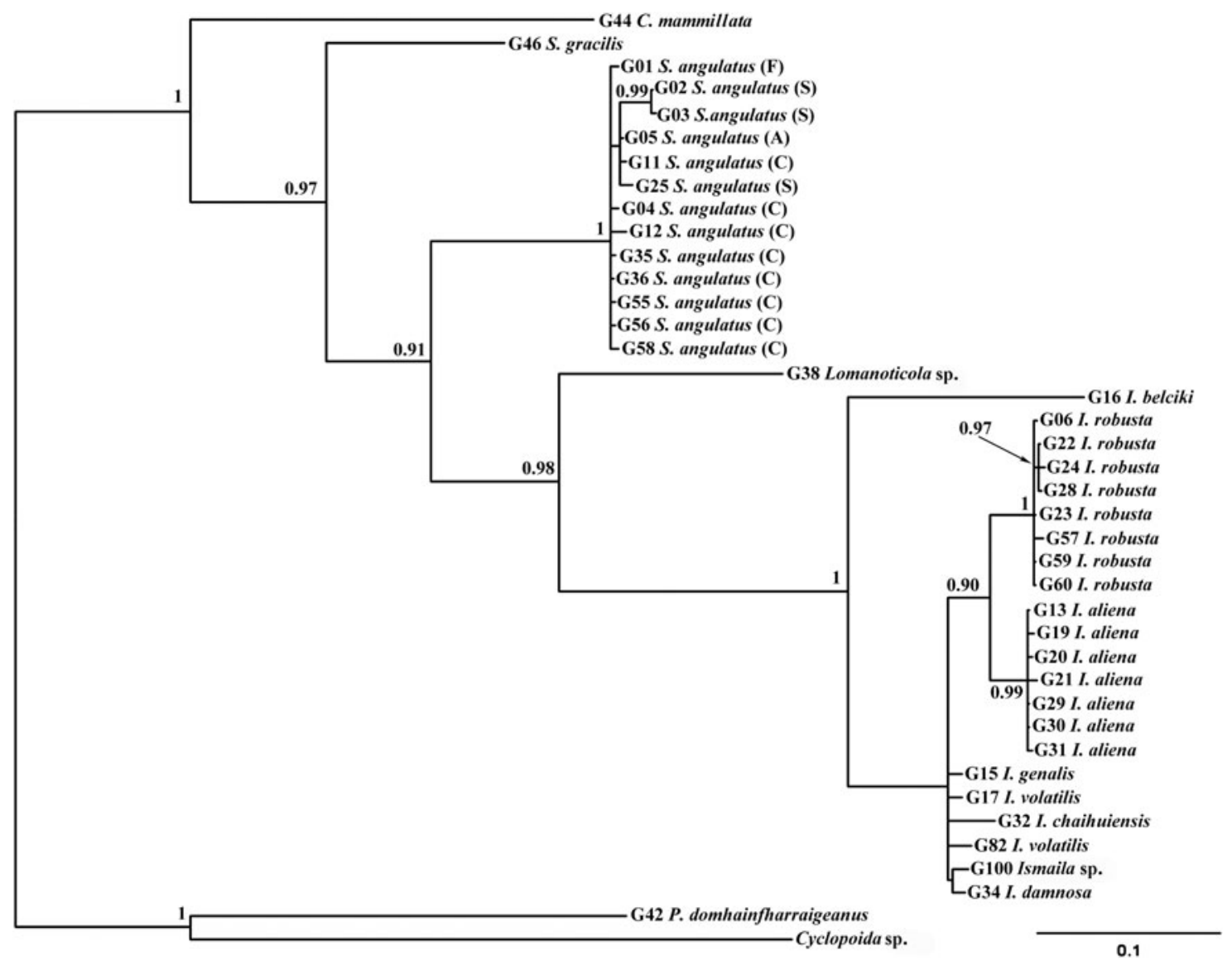

Fig. 4. Bayesian inference consensus tree of the cytochrome c oxidase I (COI) sequences of 38 splanchnotrophids and two outgroup taxa. Numbers above branches show posterior probability of BI (>0.90); branch length indicates substitutions per site. Capitals in parentheses refer to respective hosts: C: Cratena peregrina; $\mathrm{S}$ : Spurilla neapolitana; F: Flabellina ischitana; A: Aeolidiella alderi.

congruent regarding the $\mathrm{ML}$ and $\mathrm{BI}$ approach implemented in the PTP-analysis. Differences include the clade containing Ismaila sp. emerging as one species in the ML approach, while I. genalis, I. chaihuiensis and one sequence of I. volatilis (G17) are recovered as distinct species in the BI approach (see Figure $5 \mathrm{~F}$ ).

\section{Haplotype networks}

Each of the $13 \mathrm{~S}$. angulatus sequences represented a distinct haplotype. The analysis using TCS software with a 90\% statistical parsimony connection limit led to one network linking all haplotypes. In this network the inferred ancestral haplotype was from the host Cratena peregrina. Other haplotypes from this host were connected nearby (except $\mathrm{G}_{4}$ and $\mathrm{G}_{12}$ ), whereas those infesting other host species occupied more derived positions (Figure 6). However, setting the statistical parsimony connection limit to $95 \%$, as is usually applied, resulted in three separate networks (see Figure 7). The first consisted of two sequences from the host Cratena peregrina and the second consisted of the two haplotypes Go2 and Go3 (infesting Spurilla neapolitana). The third network comprised the rest of sequences, with all sequences from haplotypes infesting Cratena peregrina inferred to be more ancestral and the haplotypes of three specimens infesting other hosts occurred in the more derived positions (Figure 7).
For the genus Ismaila, i.e. I. belciki, I. aliena, I. genalis and I. chaihuiensis were recovered as independent networks under a $95 \%$ statistical parsimony connection limit. Although most haplotypes of $I$. robusta emerged as a single network, there were two haplotypes (Go6 and G59) that separated into an independent haplotype network. Another independent network consisted of a single haplotype shared by I. volatilis, Ismaila sp. and I. damnosa. However the second included haplotype of I. volatilis formed a separate network (Figure 8).

\section{Diagnostic nucleotides}

Splanchnotrophus gracilis differed from S. angulatus in 81 single pure characters (following Sarkar et al., 2008; Jörger \& Schrödl, 2014). Lomanoticola sp. differed from the genus Splanchnotrophus in 40 single pure characters. Within the genus Ismaila, I. belciki showed the highest divergence with 31 single pure characters differing from other Ismaila species. Ismaila robusta differed in nine, I. aliena in six, $I$. chaihuiensis in five, I. genalis in four and I. damnosa, Ismaila sp. and I. volatilis in one single pure character respectively.

Inside $S$. angulatus there were no differing single pure characters discernable; however, those parasites extracted from the host $C$. peregrina differed in nine single private characters from those infesting other host species. In addition, the $S$. 

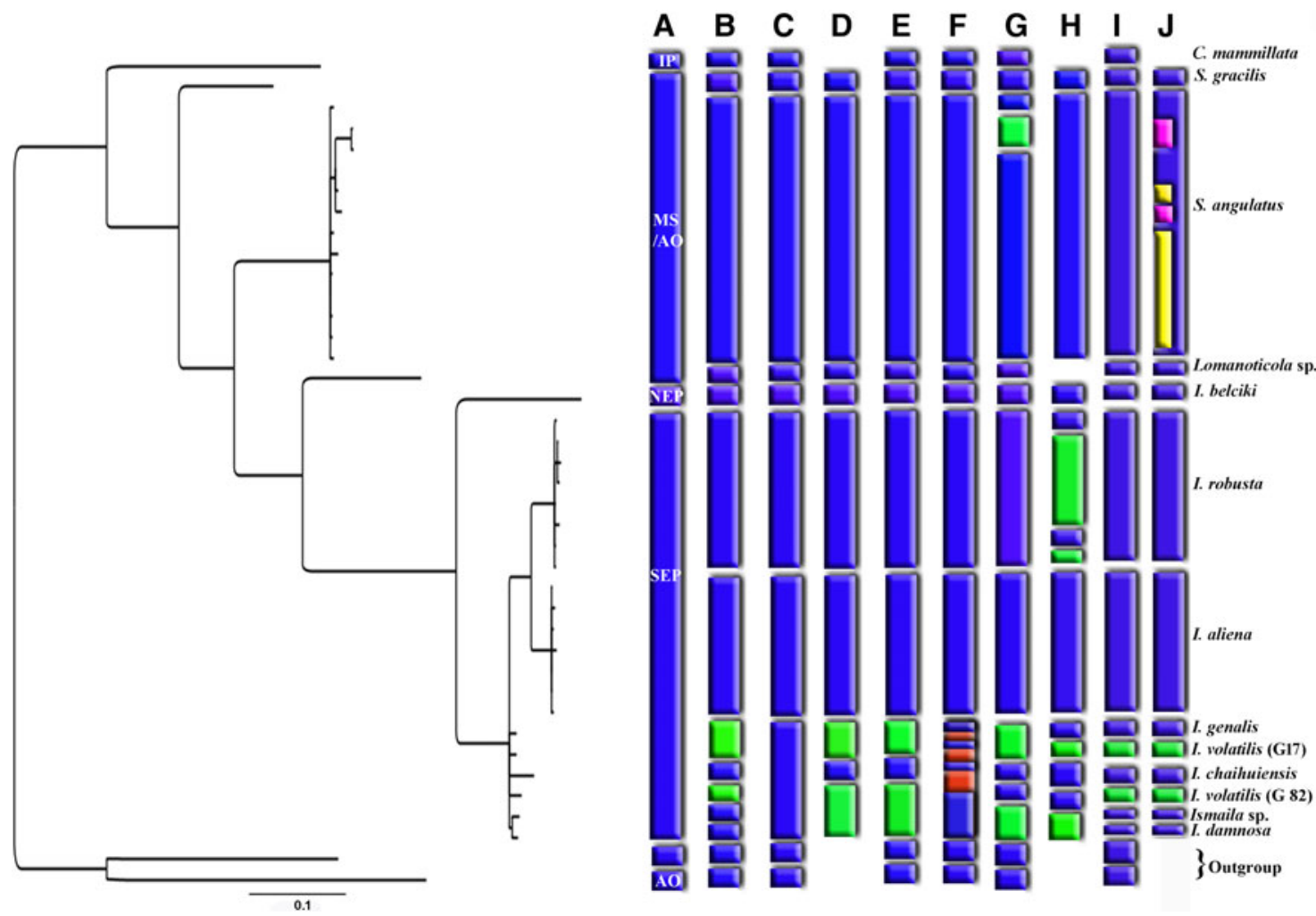

Fig. 5. Geographic distribution, sequence clusters and potential species obtained with the respective methods plotted in the Bayesian Inference tree. (A) Geographic distribution: IP, Indo-Pacific; MS, Mediterranean Sea; AO, Atlantic Ocean; NEP, north-eastern Pacific; SEP, south-eastern Pacific; (B) Maximum likelihood; (C) Bayesian inference; (D) ABGD; (E) Species Identifier; (F) PhyloMap-Poisson Tree Processes (PTP). The blue bars represent congruent results of the ML/BI approach, while the red bar indicates the differing results of the ML/BI approach; $(G)$ SPLiTstreE; (H) Tcs independent parsimony haplotype networks; (I) traditional species hypotheses based on morphological characters; (K) diagnostic nucleotides. Bars represent clades. Green bars represent clades in the respective analysis, which are not represented in the Bayesian Inference tree. Yellow and pink bars indicate groups within $S$. angulatus infesting $S$. neapolitana (pink) and C. peregrina (yellow) differing only in single private characters.

angulatus found in S. neapolitana also differed in nine single private characters from all other conspecifics. These nine single private characters did not overlap.

\section{DISCUSSION}

The high species diversity of copepods makes morphological identification and quantification of species a challenging task (Blanco-Berical et al., 2014). In such cases DNA barcoding can be a simple but suitable tool to help identify species and to shed at least some light at the respective relationships (Blanco-Berical et al., 2014; Jörger et al., 2014; Padula et al., 2014). However, barcoding identification requires that the taxonomy of the group is known, and that these taxonomic units correspond to a clade of COI sequences. This is the first attempt to apply molecular techniques to members of the Splanchnotrophidae to test the current morphologybased species hypotheses and to study the host specificity of selected members of the family.

\section{Phylogeny of the Splanchnotrophidae}

The resulting molecular trees are generally congruent with the current morphocladistic hypotheses on splanchnotrophid phylogeny (Anton \& Schrödl, 2013a, b). The traditionally accepted monophyly of Splanchnotrophidae (e.g. Huys, 2001) is supported here, as is the monophyly of the
Panamerican genus Ismaila. Splanchnotrophus, another morphology-based genus represented herein with multiple individuals, appeared paraphyletic. Surprisingly, S. gracilis, infesting the dorid nudibranch Trapania tartanella (Ihering, 1886), was recovered sister to all splanchnotrophids but Ceratosomicola. The COI topologies (Figures 3 \& 4) suggested Ceratosomicola as earliest splanchnotrophid offshoot, which is also in accord with the results of the morphocladistic analyses of Anton \& Schrödl (2013a, b). Interestingly, Ismaila is sister to Lomanoticola in the molecular trees, while morphological data usually suggested a clade of Splanchnotrophus and Lomanoticola. This supports Huys (2001) who elevated Lomanoticola, which was previously considered a subgenus of Splanchnotrophus (Hecht, 1895; Monod \& Dollfus, 1932; Delamare Deboutteville, 1950; Jensen, 1990), to genus rank. Obviously, future molecular analyses should include further splanchnotrophid species, covering the entire generic, morphological and geographic diversity of the family, and representatives of Briarella, the putative sister of Splanchnotrophidae. As indicated by high support values, the barcoding fragment of COI appears informative for resolving splanchnotrophid genus level phylogeny.

On a species level, molecular phylogenetic trees are compatible with traditional taxonomy, but do not resolve all of the valid parasite species based on morphology. COI trees confirm the monophyly of $S$. angulatus and its separation from S. gracilis (Figures 2-5) as already suggested by previous studies based on morphological data (Huys, 2001; Abad et al., 


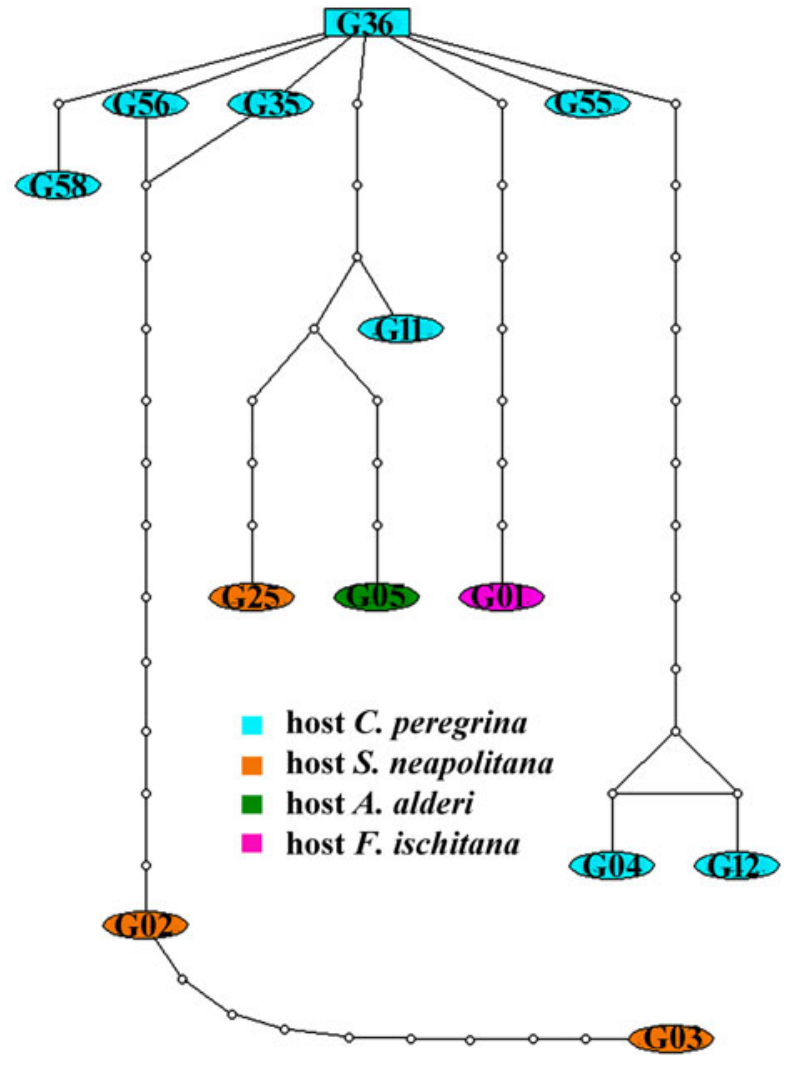

Fig. 6. Statistical parsimony network of 13 COI haplotypes in Splanchnotrophus angulatus with a connection limit set to $90 \%$; white dots represent intermediate haplotypes missing in the sample set.

2011; Anton \& Schrödl, 2013a, b). Within Ismaila, the morphologically clearly distinct species $I$. robusta, I. aliena and I. belciki were recovered monophyletic, while the recently described and similarly characteristic I. volatilis was not. The remaining species I. genalis, I. chaihuiensis, Ismaila sp. and $I$. damnosa emerged as a common clade in the ML analysis but paraphyletic in the BI analysis.

Phylogenetic trees showing a characteristic branching pattern with long internodes leading to well-supported shallow nodes with a couple of short terminals are often

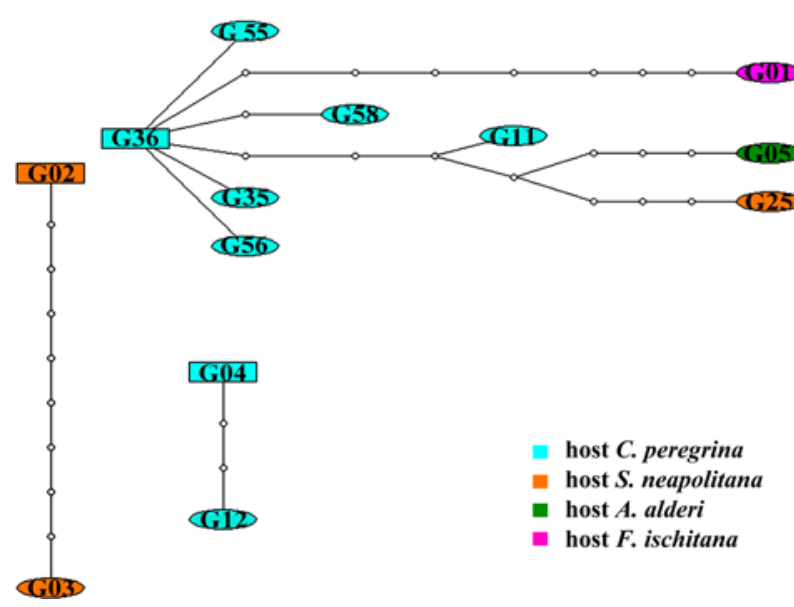

Fig. 7. Statistical parsimony network of 13 COI haplotypes in Splanchnotrophus angulatus with a connection limit of $95 \%$; white dots represent intermediate haplotypes missing in the sample set.
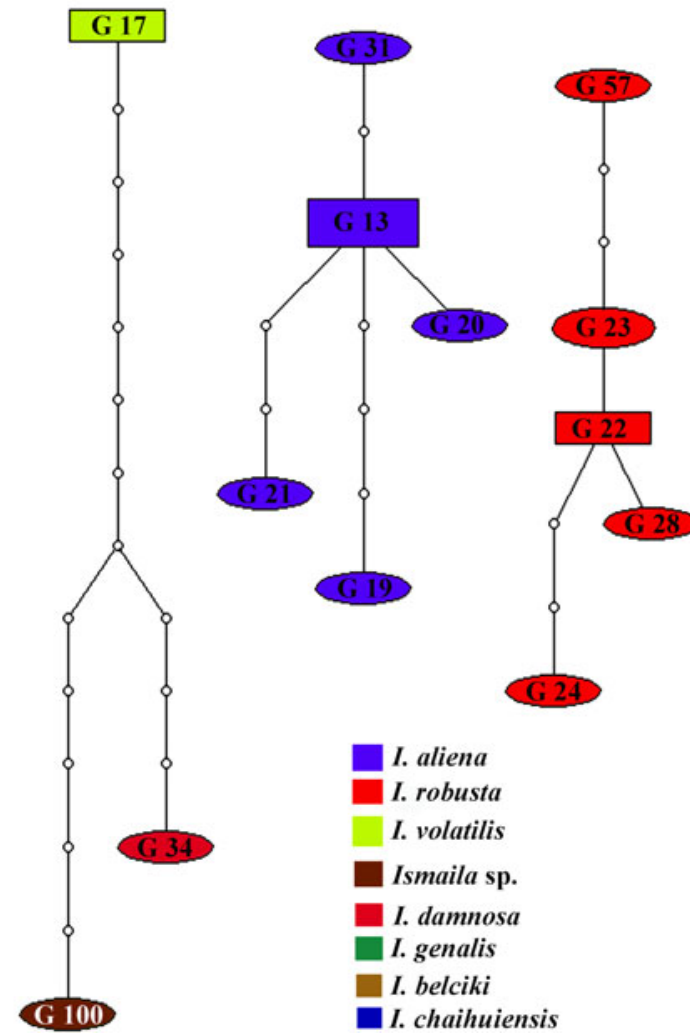

Fig. 8. Statistical parsimony network of 19 haplotypes of the genus Ismaila with a connection limit of 95\%; white dots represent intermediate haplotypes missing in the sample set.

believed to be suggestive for species units, although there is no objective way to interpret the meaning of such units and their potential substructure appropriately by eye. In current barcoding practice, even a distance-based, quickly calculated COI genealogy, combined with some genetic threshold value, may deliver a first approximation on potential species (e.g. Layton et al., 2014), and this may be useful to get a rough estimate on species diversity, e.g. when dealing with rare(ly sampled) groups or remote habitats (Jörger et al., 2010, 2014; Padula et al., 2014). However, gene histories may differ, and splanchnotrophid species level relationships appear to be complicated. Our initial phylogenetic, species delimitation and network analyses herein are based on a single gene and on an incomplete taxon and population sampling, and are inevitably preliminary.

\section{Molecular species delimitation}

Regarding Splanchnotrophus, both SPECIES IDENTIFIER and ABGD basically confirmed the two morphological species $S$. gracilis and $S$. angulatus (Figure 5), showing considerable minimum interspecific p-distance of $16.4 \%$. This is also supported by the presence of 81 single pure diagnostic characters and the results of the PTP-analysis (Figure ${ }_{5} \mathrm{~F}$ ). However, two of the three Spurilla infesting $S$. angulatus animals isolated from the same host individual were separated under certain ABGD permutations. The hypothesis of a third, 
morphologically cryptic Splanchnotrophus species is supported by the haplotype network analysis (Figures 6-8), since recovering separate networks using a $95 \%$ connection limit is sometimes used as a predictor of speciation; e.g. Miralles et al. (2011) considered species as distinct if showing separate mtDNA haplotype networks and unshared nDNA haplotypes. Unfortunately there are no reliable data from nuclear markers available for the Splanchnotrophidae.

According to the presence and number of diagnostic nucleotides both $S$. gracilis and Lomanoticola sp. receive good support. Regarding Ismaila, I. belciki is clearly separated from $I$. robusta and I. aliena also supported by differences in 31 single pure diagnostic characters. Within the genus, however, there is only poor support for the included species regarding diagnostic nucleotides. With a maximum of nine single pure characters $I$. robusta gains the highest support, but I. damnosa, Ismaila sp. and I. volatilis differ only in one single pure character respectively. Regarding S. angulatus there are no differences in single pure characters detectable according to the respective host species, supporting the hypothesis of one species displaying a lower level of host specificity. However the nine independent single private characters found for those individuals infesting $S$. neapolitana and those infesting C. peregrina respectively seem to indicate some kind of autocorrelation between gene flow and host.

In contrast to the ambiguous phylogenetic analyses, ABGD indicates I. chaihuiensis as a distinct species also (Figure $5 \mathrm{D}$ ).

Ismaila aliena, I. chaihuiensis, I. belciki and I. genalis are supported as distinct species by the results of the TCS analysis (Figures $5 \mathrm{H}-8$ ), since they all were recovered as independent networks or independent haplotypes, respectively. Ismaila robusta is also supported, nevertheless two sequences emerged as independent haplotypes (Figure $5 \mathrm{H}$ ). In the case of Go6 a possible explanation for this separation could be the geographic origin of the sample, which is quite distant to the location of all the other samples of I. robusta (see Table 1). G59, however, was collected in the same location as the rest of the specimens, so the separation from the other haplotypes remains unexplained. Neither changing the connection limit nor excluding any other haplotype had any influence on the result. The large number of inferred extinct or unsampled haplotypes suggests the data set is highly undersampled, which can result in inferring more structure than is actually present.

Ismaila damnosa, Ismaila sp. and I. volatilis emerging in the same haplotype network might initially seem to contradict the hypothesis of independent species. However, these three species are each represented only by a single sequence, rendering any attempt of estimating the intra- or interspecific variation impossible. Only a single pure diagnostic character supports these three species respectively, but this may also change as data increase. At the present time, at least some diagnostic nucleotides were found for all included Ismaila species; future exploration of the quantity and significance of diagnostic characters needs more genetic material, and the validity of these species remains somewhat equivocal.

\section{Host specificity: Ismaila versus Splanchnotrophus}

Of the morphology-defined Ismaila species included in the molecular analyses, the specific status of I. aliena and $I$. robusta was unambiguously confirmed. Both Ismaila aliena and I. robusta were previously assumed to be strictly host specific (to the dorid nudibranchs Thecacera darwini Pruvot-Fol, 1950 and Okenia luna Millen, Schrödl, Vargas \& Indacochea, 1994, repectively), and this is supported herein. Assessing the specificity of the remaining Ismaila species is much harder since there are so few observations. The limited barcoding data to date remains compatible with assuming strict host specificity of the herein included I. belciki, I. damnosa, I. genalis, I. volatilis, I. chaihuiensis and Ismaila sp. (Figure 5 ). This null hypothesis of specificity was generated by the state being plesiomorphic in the phylogenetic hypothesis of Anton \& Schrödl (2013a), and in light of our initial molecular data, there is no reason yet to assume host-induced morphological plasticity in Ismaila. We conclude that the earlier hypothesis of a species-rich neotropical clade Ismaila showing a rather rapid and recent radiation via host switches (Schrödl, 2003; Anton \& Schrödl, 2013a, b) remains a plausible evolutionary scenario.

Splanchnotrophus angulatus was recovered as a single species in both phylogenetic analyses (Figures $3 \& 4$ ). There is no genetic substructure suggestive of a hidden species complex according to the ABGD analysis, which showed no distinct barcode gap for $S$. angulatus. In the light of barcoding data, $S$. angulatus is a single species infesting various host species, including the aeolids Spurilla neapolitana, Aeolidia alderi, Cratena peregrina and Flabellina ischitana, comprising three different host families. Interestingly, two of three members of $S$. angulatus infesting Spurilla neapolitana cluster together in both phylogenetic analyses. This subgroup is also supported by the results of the ABDG- and TCS analyses (Figures 6-8). This genetically derived group may reflect some reproductive isolation due to distinct host species and represents a beginning state of speciation. According to the results of the TCS analysis there is also another group separating from the rest, consisting of two haplotypes infesting Cratena peregrina. Nevertheless, divergences are low, ABGD analyses show no distinct barcode gap, and only single private characters were found, suggestive of early divergence or limited gene flow due to ecological host differences. Morphological comparisons thus are overdue to scrutinize current taxonomy, and they need to be on a broader basis, i.e. revising all relevant Splanchnotrophus type material and specimens from a broad range of hosts.

\section{The different life-history strategies and their potential reasons}

All members of the Splanchnotrophidae capable of infesting more than two host species were reported from the Mediterranean Sea and the European coasts of the Atlantic Ocean (Figure 1), and all belong to the genus Splanchnotrophus in a broad sense. Huys (2001) split Lomanoticola from Splanchnotrophus, and both were considered either sister taxa or Splanchnotrophus deriving from paraphyletic Lomanoticola (Anton \& Schrödl, 2013a). Regardless, the ability to infest several, not necessarily closely related hosts, appeared phylogenetically and geographically correlated. Morphocladistic and molecular tree hypotheses all support a scenario in which ancestral splanchnotrophid lineages, Ceratosomicola, Ismaila and Arthurius are highly specific to a single host. Assuming diversification via 
host switch in Ismaila (Anton \& Schrödl, 2013a, b), infestation of a new host seems to invariably reduce or lose the ability to infest the original host, thus creating a bottleneck leading to a reproductive barrier. An obvious consequence of this scenario, if confirmed, is that strictly host-specific lineages can radiate in sympatry, adapting to different hosts. Strict dependence on certain sea slug hosts, which may be highly sporadic or rare (Schrödl, 2003), means higher risk of rapid extinction of newly diversified parasites. In contrast, host-promiscuous Lomanoticola and Splanchnotrophus, if confirmed by morphology-based taxonomy, may need allopatry to diverge permanently, and would have a lowered extinction risk.

\section{CONCLUSION}

The present study successfully extracted genetic material from the egg sacs of female parasites, with minimal damage of rare specimens (Anton et al., 2013). Our preliminary molecular study on splanchnotrophids included 11 of the currently 32 known species and a new Ismaila sp., many with single or few specimens; the need for more samples and markers thus is obvious. These first molecular-based analyses are largely but not fully congruent with morphology-based taxonomic hypotheses on Splanchnotrophidae (Figure 5). In addition, host specificity reported Splanchnotrophus, could be confirmed. Amphi-American Ismaila appears to radiate via host switches, losing connection to ancient populations, while individuals of Splanchnotrophus angulatus infesting different hosts may maintain some gene exchange. Uncovering details, reasons and consequences of these substantially different ecological and evolutionary strategies in the family Splanchnotrophidae provides an interesting field of research. In addition to morphology-based taxonomic revisions, we need more information on the life cycles of splanchnotrophids, on mechanisms of infections and on population dynamics of parasites and hosts to understand coevolution.

\section{ACKNDWLEDGEMENTS}

Our gratitude goes to Katharina Jörger (LMU), Tanja Korshunova and Alexander Martynov (Moscow State University), Roland Meyer, Roland Melzer, Vinicius Padula, Andrea Salmen and Enrico Schwabe (all ZSM) for providing specimens for the analyses. Thanks go to Katharina Jörger and Isabella Stöger (ZSM) for their assistance with the DNA-processing and analysis.

\section{FINANCIAL SUPPDRT}

The study and the collection trip to southern Chile were financed by a graduate scholarship of the Universität of Bayern e.V. and the GeoBioCenter LMU/Germany. Further support came from the German Research Foundation (SCHR667/13-1 and SCHR667/15-1).

\section{CONFLICT OFINTEREST}

None.

\section{REFERENCES}

Abad M., Díaz-Agras G. and Urgorri V. (2011) Anatomical description and biology of the Splanchnotrophid Splanchnotrophus gracilis Hancock \& Norman, 1863 found parasitizing the Doridacean Nudibranch Trapania tartanella Ihering, 1886 at the Ría de Ferrol (Galicia, NW Iberian Peninsula). Thalassas 27, 49-6o.

Anton R.F., Schories D., Joerger K.M., Kaligis F. and Schrödl M. (2015) Description of four new endoparasitic species of the family Splanchnotrophidae (Copepoda, Poecilostomatoida) from nudibranch and sacoglossan gastropod hosts. Marine Biodiversity 46, 183-195.

Anton R.F. and Schrödl M. (2013a) The gastropod - crustacean connection: towards the phylogeny and evolution of the parasitic copepod family Splanchnotrophidae. Zoological Journal of the Linnean Society $167,501-530$

Anton R.F. and Schrödl M. (2013b) The "inner values" of an endoparasitic copepod - computer-based ${ }_{3} \mathrm{D}$-reconstruction of Ismaila aliena. Spixiana 36, 183-199.

Anton R.F., Stevenson A. and Schwabe E. (2013) Description of a new abyssal copepod associated with the echinoid Sperosoma grimaldii Koehler, 1897. Spixiana 36, 201-210.

Bassett-Smith P.W. (1903) On new parasitic Copepoda from Zanzibar and East Africa, collected by Mr. Cyril Crossland, B. A., B. Sc. Proceedings of the Zoological Society London 73, 104-107.

Blanco-Berical L., Cornils A., Copley N. and Bucklin A. (2014) DNA barcoding of marine copepods: assessment of analytical approaches to species identification. PLOS Currents Tree of Life 6. doi: 10.1371/

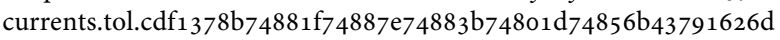
43791622 .

Canu E. (1891) Sur quelques Copépodes parasites, observés dans le Boulonnais. Comptes Rendus Hebdomadaire des Séances de íAcademie des Sciences, Paris 113, 435-437.

Carmona L., Lei B.R., Pola M., Gosliner T.M., Valdés Á and Cervera J.L. (2014) Untangling the Spurilla neapolitana (Delle Chiaje, 1841) species complex: a review of the genus Spurilla Bergh, 1864 (Mollusca: Nudibranchia: Aeolidiidae). Zoological Journal of the Linnean Society 170, 132-154.

Castresana J. (2000) Selection of conserved blocks from multiple alignments for their use in phylogenetic analysis. Molecular Biology and Evolution 17, 540-552.

Clement M., Posada D. and Crandall K.A. (2009) TCS: a computer program to estimate gene genealogies. Molecular Ecology 9, 1657-1659.

Delamare Deboutteville C. (1950) Contribution a la conaissance des Copepodes genre Splanchnotrophus Hancock \& Norman parasites de mollusques. Vie et Milieu 1, 74-80.

Folmer O., Black W., Hoeh W., Lutz R. and Vrijenhoek R. (1994) DNA primers for amplification of mitochondrial cytochrome $\mathrm{c}$ oxidase subunit I from diverse metazoan invertebrates. Molecular Marine Biology and Biotechnology 3, 294-299.

Gotto R.V. (1979) The association of copepods with marine invertebrates. Advances in Marine Biology 16, 1-109.

Gotto R.V. (2004) Commensal and parasitic copepods associated with marine invertebrates. Shrewsbury: Field Studies Council.

Hall T.A. (1999) BioEdit: a user-friendly biological sequence alignment editor and analysis program for Windows 95/98/NT. Nucleic Acids Symposium Series 41, 95-98.

Haumayr U. and Schrödl M. (2003) Revision of the endoparasitic copepod genus Ismaila Bergh, 1867, with description of eight new species. Spixiana 26, 1-33. 
Hecht E. (1895) Contributions a létude des Nudibranches. Mémoires de la Société Zoologique de France 8, 539-711.

Ho J.S. (2001) Why do symbiotic copepods matter? Hydrobiologia $453 / 454,1-7$.

Huson D.H. and Bryant D. (2006) Application of phylogenetic networks in evolutionary studies. Molecular Biology and Evolution 23, 254-267.

Huys R. (2001) Splanchnotrophid systematics, a case of polyphyly and taxonomic myopia. Journal of Crustacean Biology 21, 106-156.

Jensen K.R. (1990) Splanchnotrophus elysiae n. sp. (Copepoda; Splanchnotrophidae) found parasitizing in the sacoglossan opisthobranch Elysia australis (Quoy and Gaymard, 1832). In Proceedings of the Third International Marine Biological Workshop: The Marine Flora and Fauna of Albany, Western Australia - Western Australia Museum, Perth, Volume 1, pp. 291-296.

Jörger K., Neusser T.P., Brenzinger B. and Schrödl M. (2014) Exploring the diversity of mesopsammic gastropods: How to collect, identify, and delimitate small and elusive sea slugs? American Malacological Bulletin 32, 290-307.

Jörger K. and Schrödl M. (2014) How to use CAOS software for taxonomy? A quick guide to extract diagnostic nucleotides or amino acids for species descriptions. Spixiana 37, 21-26.

Jörger K.M., Stöger I., Kano Y., Fukuda H., Knebelsberger T. and Schrödl M. (2010) On the origin of Acochlidia and other enigmatic euthyneuran gastropods, with implications for the systematics of Heterobranchia. BMC Evolutionary Biology 10, 323. doi: 310.1186/ 1471-2148-1110-1323.

Layton K.K., Martel A.L. and Hebert P.D. (2014) Patterns of DNA barcode variation in Canadian marine molluscs. PloS ONE 9, e95003.

Meier R., Shiyang K., Vaidya G. and Ng P.L.K. (2006) DNA barcoding and taxonomy in Diptera: a tale of high intraspecific variability and low identification success. Systematic Biology 55, 715-728.

Miralles A., Vasconcelos R., Perera A., Harris D.J. and Carranza S. (2011) An integrative taxonomic revision of the Cape Verdean skinks (Squamata, Scincidae). Zoologica Scripta 40, 16-44.

Monod T. and Dollfus R.-P. (1932) Les Copépodes parasites de mollusques. Annales de Parasitologie humaine et comparée 10, 129-204.

O'Donoghue C.H. (1924) Report on Opisthobranchiata from the Abrolhos Islands, Western Australia, with description of a new parasitic copepod. Journal of the Linnean Society (Zoology) XXXV, 521-579.

Padula V., Araújo A.K., Mathews-Cascon H. and Schrödl M. (2014) Is the Mediterranean nudibranch Cratena peregrina (Gmelin, 1791) present on the Brazilian coast? Integrative species delimitation and description of Cratena minor n. sp. Journal of Molluscan Studies 80, 575-584.

Puillandre N., Lambert A., Brouillet S. and Achaz G. (2011) ABGD, Automatic Barcode Gap Discovery for primary species delimitation. Molecular Ecology. doi: 10.1111/j.1365-294X.2011.05239.x.

Puillandre N., Modica M.V., Zhang Y., Sirovich L., Boisselier M.-C., Craud C., Holford M. and Samadi S. (2012) Large-scale species delimitation method for hyperdiverse groups. Molecular Ecology 21, $2671-2691$.

Ronquist F. and Huelsenbeck J.P. (2003) MrBayes 3: Bayesian phylogenetic inference under mixed models. Bioinformatics 19, 1572-1574.
Salmen A., Kaligis F., Mamangkey G.F. and Schrödl M. (2008) Arthurius bunakenensis, a new tropical Indo-Pacific species of endoparasitic copepods from a sacoglossan opisthobranch host. Spixiana 31, 199-205.

Sarkar I.N., Planet P.J. and DeSalle R. (2008) CAOS software for use in character-based DNA barcoding. Molecular Ecology Resources 8, $1256-1259$.

Schrödl M. (1997) Aspects of Chilean nudibranch biology: effects of splanchnotrophid copepod parasitism on Flabellina sp.1 (Mollusca, Nudibranchia). Opisthobranch Newsletter 23, 45-48.

Schrödl M. (2003) Sea slugs of southern South America. Hackenheim: ConchBooks.

Stamatakis A. (2014) RAxML version 8: a tool for phylogenetic analysis and post-analysis of large phylogenies. Bioinformatics 30.

Talavera G. and Castresana J. (2007) Improvement of phylogenies after removing divergent and ambiguously aligned blocks from protein sequences alignments. Systematic Biology 56, 564-577.

Tamura K., Peterson D., Peterson N., Stecher G., Nei M. and Kumar S. (2011) MEGA5: molecular evolutionary genetics analysis using maximum likelihood, evolutionary distance and maximum parsimony methods. Molecular Biology and Evolution 28, 2731-2739.

Uyeno D. and Nagasawa K. (2012) Four new species of splanchnotrophid copepods (Poecilostomatoida) parasitic on doridacean nudibranchs (Gastropoda, Opisthobranchia) from Japan, with proposition of one new genus. ZooKeys 247, 1-29.

Weis A. and Melzer R.R. (2012) How did seaspiders recolonize the Chilean fjords after glaciation? DNA barcoding of Pycnogonida, with remarks on phylogeography of Achelia assimilis (Haswell, 1885). Systematics and Biodiversity 10, 361-374.

Xia X. and Lemey P. (2009) Assessing substitution saturation with DAMBE. In Lemey P., Salemi M. and Vandamme A.-M. (eds) The phylogenetic handbook: a practical approach to DNA and protein phylogeny, 2nd edition. Cambridge: Cambridge University Press, pp. $615-630$.

Xia X., Xie Z., Salemi M., Chen L. and Wang Y. (2003) An index of substitution saturation and its application. Molecular Phylogenetics and Evolution 26, 1-7.

Yoshikoshi K. (1975) On the structure and function of the alimentary canal of Tigriopus japonicus (Copepoda; Hapacticoida) - I. Histological structure. Bulletin of the Japanese Society of Scientific Fisheries 41, 929-935.

and

Zhang J., Kapli P., Pavlidis P. and Stamatakis A. (2013) A general species delimitation method with applications to phylogenetic placements. Bioinformatics 29, 2869-2876.

\section{Correspondence should be addressed to:}

R.F. Anton

Mollusca Department, SNSB-Bavarian State Collection of Zoology Munich, Münchhausenstraße 21, D-81247 München, Germany email: rolandanton1@gmail.com 